\title{
Regulation of the expression of claudin 23 by the enhancer of zeste 2 polycomb group protein in colorectal cancer
}

\author{
NATALIA MARYAN $^{1 *}$, MALGORZATA STATKIEWICZ ${ }^{2 *}$, MICHAL MIKULA $^{2}$, KRZYSZTOF GORYCA $^{2}$, \\ AGNIESZKA PAZIEWSKA ${ }^{1}$, ADRIANA STRZAŁKOWSKA ${ }^{2}$, MICHALINA DABROWSKA ${ }^{2}$, \\ MATEUSZ BUJKO ${ }^{3}$ and JERZY OSTROWSKI ${ }^{1,2}$ \\ ${ }^{1}$ Department of Gastroenterology and Hepatology, Medical Center for Postgraduate Education; \\ Departments of ${ }^{2}$ Genetics, and ${ }^{3}$ Molecular and Translational Oncology, \\ Maria Sklodowska-Curie Memorial Cancer Center and Institute of Oncology, Warsaw 02-781, Poland
}

Received April 27, 2014; Accepted January 21, 2015

DOI: $10.3892 / \mathrm{mmr} .2015 .3378$

\begin{abstract}
Altered epigenetic mechanisms, similar to gene mutations, contribute to the pathogenesis and molecular heterogeneity of neoplasms, including colorectal cancer (CRC). Enhancer of zeste 2 (EZH2) is a histone methyltransferase, which is involved in epigenetic gene silencing and is aberrantly expressed in CRC. Therefore, the identification of the genes regulated by EZH2 in CRC is important to improve current understanding of its role in cancer epigenetics. The present study used chromatin immunoprecipitation (ChIP) followed by deep sequencing to assess genome-wide EZH2-DNA interactions in healthy or CRC mucosa samples. In total, 86.9/61.6 and 92.5/62.6 million tags were sequenced/mapped in healthy and CRC mucosa samples, respectively. The EZH2-binding densities were correlated with transcriptomic datasets and this demonstrated that the claudin-23 (CLDN23) gene, which encodes a component of cell-cell adhesion structures, was occupied by EZH2 and significantly silenced in CRC tissue. The measurement of DNA methylation at the CLDN23 promoter using pyrosequencing excluded the possibility that silencing of this gene in CRC patient samples was a result of DNA hypermethylation. Following treatment of the Colo205 and HT-29 CRC cell lines, with the EZH2 inhibitor, GSK126, the level of histone H3 lysine 27 trimethylation (H3K27me3) was reduced and the mRNA and protein expression levels of CLDN23 were increased. ChIP analysis confirmed that the level of $\mathrm{H} 3 \mathrm{~K} 27 \mathrm{~m} 3$ along the CLDN23 gene was decreased in
\end{abstract}

Correspondence to: Professor Jerzy Ostrowski, Maria Sklodowska-Curie Memorial Cancer Center and Institute of Oncology, Roentgena 5, Warsaw 02-781, Poland

E-mail: jostrow@warman.com.pl

${ }^{*}$ Contributed equally

Key words: colorectal cancer, ChIP-Seq, enhancer of zeste 2, claudin 23, GSK126 the GSK126-treated cell lines. Furthermore, ChIP analysis of these samples detected histone $\mathrm{H} 3$ lysine 4 trimethylation (H3K4me3) at the CLDN23 promoter, demonstrating that the balance between $\mathrm{H} 3 \mathrm{~K} 27 \mathrm{me} 3$ and $\mathrm{H} 3 \mathrm{~K} 4 \mathrm{me} 3$ may underlie the regulation of the expression of CLDN23. The present study demonstrated an epigenetic link between the activity of the EZH2 methyltransferase at the CLDN23 locus and the expression of CLDN23 in CRC tissue.

\section{Introduction}

Gene expression is regulated at different hierarchical levels, one of which is the accessibility of genes and their cis-regulatory elements to the transcriptional complex. This can be effected by the degree of chromatin compaction, which is controlled, in part, by epigenetic regulators belonging to the polycomb group of proteins (1). The histone methyltransferase, enhancer of zeste 2 (EZH2), is a component of the polycomb-repressive complex (PRC) 2, which catalyzes the methylation of histone $\mathrm{H} 3$ lysine 27 residues and mediates the epigenetic silencing of target genes. Identification of the genes that PRC2 regulates is important as EZH2 is often aberrantly expressed in neoplasms, including colorectal cancer (CRC) (2).

Cell-to-cell adhesion in the epithelial cell monolayer is mediated by four types of junctions: Tight junctions, adherens junctions, gap junctions and desmosomes (3). Claudins (CLDNs) constitute the backbone of tight junctions and are comprised of a multigene family of 24 protein members with molecular masses ranging between 20 and $27 \mathrm{kDa}$. The CLDN proteins create paracellular barriers, which are critical for sealing epithelial sheets, maintaining cell polarity and controlling the flux of ions and small molecules between epithelial cells (4). These proteins also regulate intercellular signaling and cell morphology (5). CLDNs are expressed in a tissue-specific manner and the majority of tissues express multiple CLDNs (6). Aberrant expression of CLDNs has been demonstrated in several malignancies and this is dependent on the tumor localization, stage and grade $(4,7)$. However, tumors exhibit changes in the expression levels of a limited number of CLDNs. Furthermore, previous studies 
have investigated the epigenetic mechanism underlying the deregulation of CLDNs in certain types of tumor $(7,8)$.

One of the characteristics of cancer is disruption of the attachment of epithelial cells to other cells and to the extracellular matrix, followed by local invasion and distant metastasis (9). E-cadherin is the predominant and most extensively investigated example of a junction component, which is involved in cancer. This protein has been implicated as a tumor suppressor in various types of cancer via its negative regulation of cancer cell invasion $(10,11)$. The importance of other protein components of intracellular junctions, including CLDNs, in tumorigenesis is also emerging. However, the molecular mechanisms underlying their altered expression in cancer and the biological significance of this remain to be elucidated (4). This is important for developing therapeutic interventions, which may restore normal intercellular attachment.

The present study demonstrated that the expression of CLDN23 is significantly reduced in CRC tissue. A chromatin immunoprecipitation followed by deep sequencing (ChIP-Seq) assay revealed that a high level of EZH2 is bound to the CLDN23 locus in CRC tissue, suggesting that $\mathrm{EZH} 2$-mediated histone methylation was responsible for the downregulation of the expression of CLDN23. The present study suggested a functional link between the binding and activity of EZH2 at the CLDN23 locus and the repression of CLDN23 expression during the development of CRC.

\section{Materials and methods}

Tissue samples. Chromatin was isolated from 12 adenocarcinoma (AC) samples and their healthy colonic mucosa (NC) counterparts, which were collected in our previous study (12). Cryostat sections were prepared from each sample using a Microm HM 505E cryostat (Zeiss, Göttingen, Germany). Upper and lower sections from each cryosection were evaluated by a pathologist to confirm whether they contained the expected cell type. Furthermore, individual RNA and DNA samples from 24 pairs of healthy colon and AC specimens, which were extracted in our previous study (13) were used for reverse transcription quantitative polymerase chain reaction (RT-qPCR) and DNA methylation analyses.

Cell lines. All cell lines were obtained from American Type Culture Collection (Rockville, MD, USA). A total of three $\mathrm{CRC}$ cell lines were cultured in a humidified $5 \% \mathrm{CO}_{2}$ atmosphere at $37^{\circ} \mathrm{C}$ in media purchased from Invitrogen Life Technologies (Carlsbad, CA, USA). The Colo205 cells were cultured in RPMI-1640 medium, supplemented with $10 \%$ fetal bovine serum (FBS; Invitrogen Life Technologies). HT-29 and HCT-116 cells were cultured in McCoy's 5A modified medium, supplemented with $10 \%$ FBS (Invitrogen Life Technologies). The cells were grown for $24 \mathrm{~h}$ in 6-well culture plates (Sarstedt AG \& Co., Nümbrecht, Germany) to $40-50 \%$ confluency, prior to treatment with dimethyl sulfoxide (0.01\%; Sigma-Aldrich, St. Louis, MO, USA) or an EZH2 inhibitor (GSK126; Biovision, Mountain View, CA, USA) at a final concentration of $0.5,1,2$ and $5 \mu \mathrm{M}$ and collected $72 \mathrm{~h}$ later. Control cells reached $80-90 \%$ confluence following $72 \mathrm{~h}$ culture.
3-(4,5-Dimethylthiazol-2-yl)-2,5-diphenyltetrazolium brom ide (MTT) assay. Following incubation with $0.5,1,2$ and $5 \mu \mathrm{M}$ GSK126 for $72 \mathrm{~h}$ in a humidified $5 \% \mathrm{CO}_{2}$ atmosphere at $37^{\circ} \mathrm{C}$, MTT solution (Sigma-Aldrich) was added at a final concentration of $0.5 \mathrm{mg} / \mathrm{ml}$ from a stock solution of $5 \mathrm{mg} / \mathrm{ml}$, prepared using phosphate-buffered saline (PBS; Sigma-Aldrich), and the cells were incubated for $3 \mathrm{~h}$ in a humidified $5 \% \mathrm{CO}_{2}$ atmosphere at $37^{\circ} \mathrm{C}$. The cells were subsequently lysed in acidified 2-propanol (POCH S.A., Gliwice, Poland) and the absorbance was subsequently measured at 550-560 nm using a Victor3 microplate reader (Perkin Elmer, Waltham, MA, USA).

ChIP assay. The tissue cryostat section $(50 \mathrm{mg}$ ) was immersed in $2 \mathrm{ml}$ hypotonic buffer $\mathrm{A}$, containing $10 \mathrm{mM}$ HEPES (pH 7.9), $2 \mathrm{mM} \mathrm{MgCl}_{2}$ and $2 \mathrm{mM} \mathrm{KCl}$, supplemented with protease and phosphatase inhibitors (78441) (Thermo Fisher Scientific, Co., Ltd., Waltham, MA, USA). The samples were ground using a loose (10 times) and tight (30 times) dounce pestle (Wheaton Industries, Inc., Millville, NJ, US) and subsequently centrifuged at $700 \mathrm{xg}$ for $5 \mathrm{~min}$ in $4^{\circ} \mathrm{C}$. The nuclei in the pellet were cross-linked in $1 \mathrm{ml}$ PBS, containing $1 \%$ formaldehyde (Sigma-Aldrich) for $10 \mathrm{~min}$ at room temperature, following which the reaction was quenched at room temperature for $5 \mathrm{~min}$ by adding glycine (Sigma-Aldrich) at a final concentration of $125 \mathrm{mM}$ (from a stock solution of $2 \mathrm{M}$ ). Following centrifugation at $700 \mathrm{x} \mathrm{g}$ for $5 \mathrm{~min}$ at room temperature, the nuclei were washed once with $1 \mathrm{ml}$ of PBS and subsequently stored at $-80^{\circ} \mathrm{C}$.

Cross-linking of the cell lines to isolate chromatin was performed, as described previously (14). Pelleted nuclei were resuspended in lysis buffer, containing $12.5 \mathrm{mM}$ Tris- $\mathrm{HCl}$ (pH 8.0), $2.5 \mathrm{mM}$ EDTA and $0.25 \%$ sodum dodecyl sulphate (SDS) (Sigma-Aldrich), supplemented with protease and phosphatase inhibitors (no. 78441; Thermo Fisher Scientific).

Chromatin was sheared in a Bioruptor Plus (Diagenode, Denville, NJ, USA) using a $30 \mathrm{sec}$ on-off cycle for $15 \mathrm{~min}$ at high intensity. ChIP assays were performed using the Matrix-ChIP method (15). Alternatively, a modified Fast-ChIP method was used for the ChIP-Seq libraries (16). ChIP DNA data are expressed as the percentage of input DNA, as described previously (14). For sequencing, ChIP was performed using a rabbit polyclonal anti-EZH2 antibody (ab3748; Abcam, Cambridge, UK) and non-specific rabbit immunoglobulin $\mathrm{G}$ (IgG; I-1000; Vector Laboratories, Burlingame, CA, USA). The following antibodies were also used in the ChIP assays: Mouse monoclonal anti-RNA polymerase II (Pol2; sc-47701; Santa Cruz Biotechnology, Inc., Santa Cruz, CA, USA), rabbit polyclonal anti-histone $\mathrm{H} 3$ lysine 27 trimethylation (H3K27m3; no. 07-449; EMD Millipore, Billerica, MA, USA), rabbit polyclonal anti-histone $\mathrm{H} 3$ (ab1791; Abcam) and rabbit polyclonal anti-histone $\mathrm{H} 3$ lysine 4 trimethylation (H3K4me3; no. 07-473; EMD Millipore).

ChIP-Seq. The DNA (10 ng) was processed using a ChIP-Seq Sample Prep Kit (IP-102-1001; Illumina, Inc., San Diego, CA, USA) according to the manufacturer's instructions, with PCR conditions for adapter-modified DNA fragments perfomed exactly as described previously (17). Deep sequencing was performed at ATLAS Biolabs GmbH (Berlin, Germany) using the Genome Analyzer IIx (Illumina, Inc.) with single-end 
$36 \mathrm{bp}$ reads. The sequences were obtained using the Analysis Pipeline (Illumina, Inc.) and then mapped to the human genome assembly (version hg19) using Bowtie software (18). The protein binding sites were detected with MACS (19), assuming a change in binding intensity of at least 10 -fold for highly confident peaks (cancerous sample, vs. healthy samples; specific antibody, vs. IgG).

Western blotting. The cells were lysed in $1 \mathrm{X}$ Laemmli buffer [65.8 mM Tris-HCl (pH 6.8), 2.1\% SDS, $26.3 \%(\mathrm{w} / \mathrm{v})$ glycerol, $0.01 \%$ bromophenol blue, $4 \% \beta$-mercaptoethanol; Sigma-Aldrich) and the proteins were resolved by SDS-PAGE and electrotransferred to polyvinylidene fluoride membranes (EMD Millipore). The blotted proteins were assessed by western blot analysis using the following antibodies: Anti-EZH2, anti-H3K27m3, anti-histone H3 and anti-CLDN23 (ab23355; Abcam).

Extraction of total RNA from the cell lines and reverse transcription. The total RNA was extracted from the cells using a TRIzol ${ }^{\circledR}$ Plus RNA Purification kit (Life Technologies) and on-column DNase I treatment was performed according to the manufacturer's instructions. The total RNA $(1 \mu \mathrm{g})$ and random hexamers were used to synthesize the cDNA with Superscript III (Life Technologies) according to the manufacturer's instructions.

$q P C R$. The qPCR of the cDNA and ChIP samples were performed using an Applied Biosystems 7900HT Fast Real-Time PCR system (Applied Biosystems Life Technologies, Foster City, CA, USA) and a Sensimix SYBR kit (Bioline Ltd., London, England) in a $5 \mu \mathrm{l}$ reaction according to manufacturers' instructions. $\alpha$-tubulin $1 \mathrm{~B}$ was used as the reference mRNA expression. The mRNA expression levels of EZH2 and CLDN23 were determined in $24 \mathrm{NC}$ and 24 AC tissue samples using RT-qPCR, as described previously (13). The sequences of the primers used are shown in Table I. Differences in expression levels were evaluated using the Mann-Whitney U-test in GraphPad Prism 5 (GraphPad Software, Inc., La Jolla, CA, USA). P<0.05 was considered to indicate a statistically significant difference.

DNA methylation analysis. The level of DNA methylation was assessed in the CRC cell lines, CRC and NC samples from 12 patients using a pyrosequencing assay. DNA was isolated using a QIAamp DNA Mini kit (Qiagen, Hilden, Germany) and bisulfite-converted using an EpiTect kit (Qiagen), according to the manufacturer's instructions. The PCR was performed in a $30 \mu \mathrm{l}$ reaction mixture, containing $1 \mathrm{X}$ PCR buffer, $2 \mathrm{mM} \mathrm{MgCl}, 0.25 \mathrm{mM}$ dNTPs, $0.2 \mu \mathrm{M}$ forward and reverse primers and 0.5 units FastStart DNA Polymerase (Roche Applied Science, Indianapolis, IN, USA). The cycling conditions were as follows: Initial denaturation at $94^{\circ} \mathrm{C}$ for $3 \mathrm{~min}$, followed by 40 cycles of $94^{\circ} \mathrm{C}$ for $30 \mathrm{sec}, 52^{\circ} \mathrm{C}$ for $30 \mathrm{sec}$ and $72^{\circ} \mathrm{C}$ for $30 \mathrm{sec}$, followed by a final elongation at $72^{\circ} \mathrm{C}$ for $7 \mathrm{~min}$. The PCR products were purified and analyzed using the PyroMark Q24 system (Biotage AB, Uppsala, Sweden), according to the manufacturer's instructions. The mean methylation levels in the amplified regions were analyzed.

\section{Results}

Genome-wide mapping of EZH2 demonstrates that the CLDN23 gene is occupied by PRC2 in the CRC samples. To identify genes, of which expression is regulated by PRC2 in CRC tissues, ChIP-Seq was performed to survey EZH2 on a pool of chromatin derived from 12 pairs of $\mathrm{NC}$ and $\mathrm{AC}$ samples. In total, 86.9/61.6 and 92.5/62.6 million tags were sequenced/mapped in the $\mathrm{NC}$ and $\mathrm{AC}$ samples, respectively. The binding densities of EZH2 were correlated with transcriptomic datasets generated in our previous microarray study of CRC samples (12). The presence of EZH2 on chromatin is often associated with chromatin compaction and transcription repression. Therefore, through this analysis, a list of genes potentially regulated by EZH2 was comprised to only include those for which the level of EZH2 binding, and the level of mRNA expression were inversely associated. As cut-offs, a 2- and 4-fold change (FC) in the EZH2-binding density and mRNA expression of a given gene, respectively, between the $\mathrm{NC}$ and AC samples was used (Table II). Among the 15 genes, which met these criteria, the expression of CLDN23 differed the most between the NC and AC samples, with a FC in mRNA expression of 7.2. This difference in the mRNA expression of CLDN23 was confirmed in 24 pairs of NC and AC cDNA samples using RT-qPCR (13). It was confirmed that, in AC tissues, the expression of CLDN23 was significantly reduced $(\mathrm{FC}=9.1)$ and the mRNA expression of $\mathrm{EZH} 2$ was markedly increased (Fig. 1).

Inspection of the EZH2 binding tracks using the UCSC genome browser (20) revealed that more EZH2 was bound to chromatin in the AC samples compared with the NC samples (Fig. 2A). ChIP-qPCR analysis of the same chromatin preparations that were used in the ChIP-Seq survey confirmed that the occupancy of the promoter and downstream regions of the CLDN23 gene by EZH2 was increased in the AC samples (Fig. 2B). In accordance with the role of EZH2 in suppressing gene expression (2), the level of $\mathrm{H} 3 \mathrm{~K} 27 \mathrm{~m} 3$ was also increased in the AC samples, while occupancy of the CLDN23 gene by the Pol 2 complex was lower in the AC samples compared with the NC samples. As a control, constitutively repressed and constitutively active gene promoters, namely, $\beta$-globin (HBB) and glyceraldehyde 3-phosphate dehydrogenase, respectively, were analyzed.

In cancer development, aberrant DNA methylation is an underlying epigenetic mechanism, which leads to the inactivation of tumor suppressor genes (9). Since the observed downregulation of the expression of CLDN23 in AC tissues may arise from DNA hypermethylation within its $\mathrm{CpG}$ island at the 5 ' end of the gene, the level of $\mathrm{CpG}$ dinucleotide methylation was measured using pyrosequencing in 12 pairs of $\mathrm{NC}$ and AC tissues. The level of cytosine methylation at the CLDN23 CpG island remained unchanged between the NC and the AC tissues (Fig. 2C). This result, together with the ChIP-based data, suggested that EZH2 affects the expression of CLDN23 in colonic tissues.

EZH2 regulates the expression of CLDN23 in colon cancer cell lines. EZH2 ChIP-Seq data acquired within the frames of the ENCODE project demonstrated that EZH2 abundantly occupied CLDN23 in several cell lines, confirming the direct 
Table I. Primers used in the present study.

\begin{tabular}{|c|c|c|c|}
\hline Gene & Sequence $\left(5^{\prime}-3\right)^{\prime}$ & Assay & Chromosome location \\
\hline \multicolumn{4}{|l|}{ RPLP0 } \\
\hline Forward & GCAATGTTGCCAGTGTCTG & \multirow{2}{*}{ RT-qPCR } & \multirow{2}{*}{ Exon $7-8$} \\
\hline Reverse & GCCTTGACCTTTTCAGCAA & & \\
\hline \multicolumn{4}{|l|}{$\mathrm{EZH} 2$} \\
\hline Forward & ATGGGAAAGTACACGGGGAT & \multirow{2}{*}{ RT-qPCR } & \multirow{2}{*}{ Exon 5-6 } \\
\hline Reverse & ATTGACCAAGGGCATTCACC & & \\
\hline \multicolumn{4}{|l|}{ CLDN23 } \\
\hline Forward & GGGTCCСТСССТССC & \multirow{2}{*}{ ChIP-qPCR } & \multirow{2}{*}{ Promoter $^{\mathrm{a}}$} \\
\hline Reverse & CTGGCCCTAATCGAAACCA & & \\
\hline \multicolumn{4}{|l|}{ CLDN23 } \\
\hline Forward & GAGGGAACTAGCCTAAGTGG & \multirow{2}{*}{ RT, ChIP-qPCR } & \multirow{2}{*}{$\mathrm{A}^{\mathrm{a}}$} \\
\hline Reverse & GAGCCCTGATCGCTCC & & \\
\hline \multicolumn{4}{|l|}{ CLDN23 } \\
\hline Forward & CAGTGGACGTGGAGTTGTA & \multirow{2}{*}{ ChIP-qPCR } & \multirow{2}{*}{$\mathrm{B}^{\mathrm{a}}$} \\
\hline Reverse & GAAGTAGCCCCACTGGTC & & \\
\hline \multicolumn{4}{|l|}{ CLDN23 } \\
\hline Forward & TCTCACTTATTTTTGCGGTGA & \multirow{2}{*}{ ChIP-qPCR } & \multirow{2}{*}{$\mathrm{C}^{\mathrm{a}}$} \\
\hline Reverse & GACCTTTGTTCTCCTCTTGG & & \\
\hline \multicolumn{4}{|l|}{ ACTB } \\
\hline Forward & ACGCCTCCGACCAGTGTT & \multirow{2}{*}{ ChIP-qPCR } & \multirow{2}{*}{ Promoter $^{\mathrm{a}}$} \\
\hline Reverse & GCCCAGATTGGGGACAAA & & \\
\hline \multicolumn{4}{|l|}{ GAPDH } \\
\hline Forward & CGCCCCCGGTTTCTATAAAT & \multirow{2}{*}{ ChIP-qPCR } & \multirow{2}{*}{ Promoter $^{\mathrm{a}}$} \\
\hline Reverse & TCGAACAGGAGGAGCAGAGAG & & \\
\hline \multicolumn{4}{|l|}{$\mathrm{HBB}$} \\
\hline Forward & GCAATAGATGGCTCTGCCCT & \multirow{2}{*}{ ChIP-qPCR } & \multirow{2}{*}{ Promoter $^{\mathrm{a}}$} \\
\hline Reverse & GACAGGTACGGCTGTCATCA & & \\
\hline \multicolumn{4}{|l|}{ CLDN23 } \\
\hline Forward & AGAAGGAAGGTAGGTTGTAGG & \multirow{2}{*}{ Pyrosequencing } & \multirow{2}{*}{ 5'-end } \\
\hline Reverse & Biotin-ACTAACTAАTTCAAAAAАCССТTCAC & & \\
\hline
\end{tabular}

involvement of EZH2 in the regulation of CLDN23 transcription (21). Based on these results, the activity of EZH2 was altered using the EZH2-specific inhibitor, GSK126, in the Colo205, HT-29 and HCT-116 colon cancer cell lines, and changes in the expression of CLDN23 were analyzed. GSK126 is a potent, highly specific small molecule inhibitor of wild-type and mutant EZH2 (22). The effects of treatment with $0.5,1,2$ and $5 \mu \mathrm{M}$ GSK126 on the viability of the colon cancer cell lines were evaluated. Treatment with $5 \mu \mathrm{M}$ GSK126 for $72 \mathrm{~h}$ was cytotoxic (Fig. 3A), whereas treatment with the lower concentrations of GSK126 were not cytotoxic to any of the cell lines. Therefore, in subsequent analyses, $2 \mu \mathrm{M}$ GSK126 was used. As demonstrated by western blotting, H3K27me3 was markedly reduced in the cell lines treated with $2 \mu \mathrm{M}$ GSK126 (Fig. 3B). To exclude the possibility that methylation of CLDN23 underlies the repression of the transcription of this gene, pyrosequencing was performed to measure the levels of methylation in a $40 \mathrm{bp}$ region of CLDN23 containing $10 \mathrm{CpG}$ dinucleotides. This revealed that the mean levels of methylation were 15.7, 26.3 and $96.8 \%$ in the HT-29, Colo205, and HCT-116 cell lines, respectively (data not shown). The level of methylation at CLDN23 was high in the HCT-116 cells, therefore, to evaluate the role of EZH2 in the repression of CLDN23 transcription, this cell line was excluded from further analyses. In accordance with transcriptional repression by EZH2, mRNA (Fig. 3C) and protein (Fig. 3D and E) expression levels of CLDN23 were increased in the HT-29 and Colo205 cell lines following treatment with $2 \mu \mathrm{M}$ GSK126.

Epigenetic changes at the CLDN23 loci are associated with GSK126 treatment. Treatment with GSK126 increased the expression of CLDN23, therefore, the epigenetic changes 
Table II. Genes exhibit increased binding of EZH2 and decreased mRNA expression levels in AC compared with NC tissues.

\begin{tabular}{|c|c|c|c|c|c|}
\hline \multirow[b]{2}{*}{ Gene } & \multicolumn{2}{|c|}{ Tag } & \multirow[b]{2}{*}{ P-value } & \multirow[b]{2}{*}{ mRNA FC AC/NC } & \multirow[b]{2}{*}{$\mathrm{FC}$ in $\mathrm{EZH} 2$ binding } \\
\hline & EZH2 AC & $\mathrm{EZH} 2 \mathrm{NC}$ & & & \\
\hline CLDN23 & 109 & 23 & $1.07^{-11}$ & 0.14 & 4.74 \\
\hline MALL & 112 & 12 & $1.67^{-10}$ & 0.16 & 9.33 \\
\hline TMEM171 & 135 & 28 & $4.30^{-10}$ & 0.17 & 4.82 \\
\hline SRPX & 108 & 20 & $7.98^{-5}$ & 0.33 & 5.40 \\
\hline LMOD1 & 118 & 22 & $6.15^{-3}$ & 0.38 & 5.36 \\
\hline CAPN5 & 130 & 21 & $9.52^{-7}$ & 0.39 & 6.19 \\
\hline SH3BGRL2 & 101 & 25 & $9.54^{-10}$ & 0.40 & 4.04 \\
\hline GLIPR2 & 139 & 27 & $1.23^{-10}$ & 0.40 & 5.15 \\
\hline CDC42SE2 & 115 & 24 & $3.80^{-7}$ & 0.43 & 4.79 \\
\hline AQP11 & 152 & 37 & $1.15^{-5}$ & 0.43 & 4.11 \\
\hline ACADVL & 112 & 18 & $1.94^{-8}$ & 0.45 & 6.22 \\
\hline WASL & 107 & 14 & $1.43^{-6}$ & 0.47 & 7.64 \\
\hline TCN2 & 107 & 23 & $2.96^{-8}$ & 0.47 & 4.65 \\
\hline C1orf106 & 132 & 21 & $5.64^{-5}$ & 0.48 & 6.29 \\
\hline GNAQ & 113 & 20 & $9.47^{-6}$ & 0.49 & 5.65 \\
\hline
\end{tabular}

FC, fold-change, expressed as the ratio of the mRNA expression in AC to NC; tag, number of reads assigned to the gene in the chromatin immunoprecipitation-sequencing dataset; $\mathrm{AC}$, adenocarcinoma; $\mathrm{NC}$, healthy surrounding mucosa negative control; EZH2, enhancer of zeste homolog 2; CLDN23, claudin23; MALL, myelin and lymphocyte-like; TMEM171, transmembrane protein 171; SRPX, sushi-repeat containing protein, X-linked; LMOD1, leiomodin 1; CAPN5, calpain 5; SH3BGRL2, SH3 domain binding glutamate-rich protein like 2; GLIPR2, GLI pathogenesis-related 2; CDC42SE2, cell division cycle 42 small effector 2; AQP11, aquaporin 11; ACADVL, acyl-coenzyme A dehydrogenase; WASL, Wiskott-Aldrich syndrome L; TCN2, transcobalamin II; C1orf106, chromosome 1 open reading frame 106; GNAQ, guanine nucleotide binding protein $\mathrm{q}$.
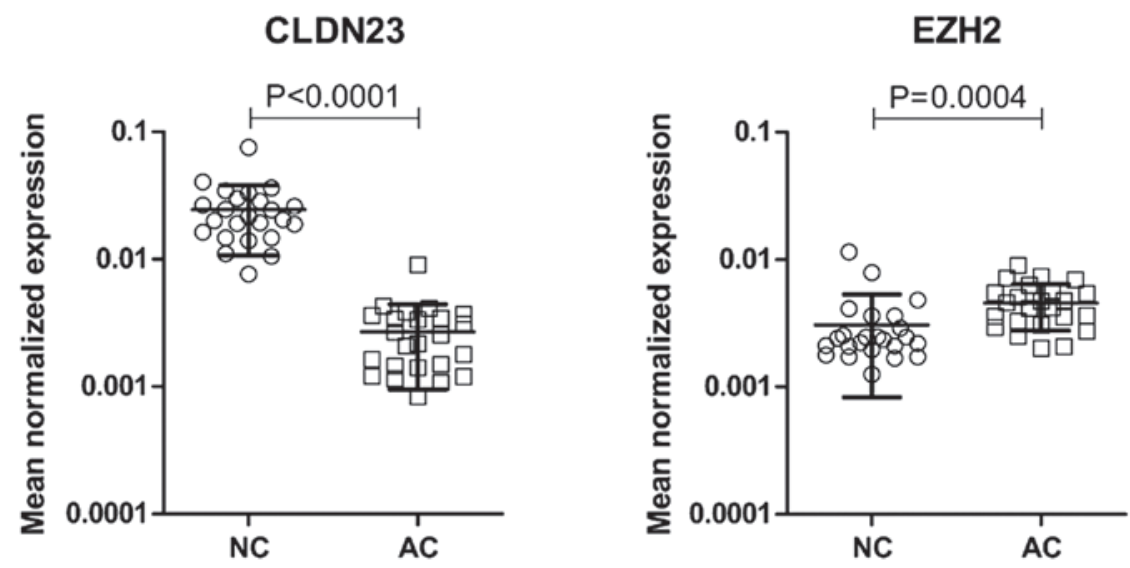

Figure 1. Analysis of the mRNA expression levels of EZH2 and CLDN23 in individual tissue samples of 24 AC and 24 NC. Horizontal bars indicate the mean and the error bars indicate the standard deviation. Differences were analyzed using the Mann-Whitney test. $\mathrm{P}<0.05$ was considered to indicate a statistically significant difference. AC, adenocarcinomas; NC, healthy mucosa; EZH2, enhancer of zeste 2; CLDN23, claudin 23.

along the CLDN23 gene, which accompany the inhibition of EZH2 in the Colo205 and HT-29 cell lines were characterized using ChIP (Fig. 4). The promoters of constitutively active and constitutively repressed genes, namely, $\beta$-actin (ACTB) and $\mathrm{HBB}$, respectively, were surveyed. As expected, in the presence of GSK126, the abundance of H3K27m3 on the CLDN23 gene decreased by 51 and $48 \%$ in the Colo205 and HT-29 cell lines, respectively. The level of H3K27m3 at the HBB promoter was also decreased in the two cell lines.
Notably, the level of $\mathrm{H} 3 \mathrm{~K} 27 \mathrm{~m} 3$ at the ACTB promoter was also decreased in the two cell lines, although to a lesser extent in the HT-29 cells compared with the Colo205 cells. ChIP analyses revealed no marked changes in the occupancy of CLDN23, the HBB promoter or the ACTB promoter by EZH2 between GSK126-treated cells and non-treated cells, indicating that GSK126 treatment does not impair the binding of EZH2. Notably, the distribution of EZH2 increased towards the 3' end of CLDN23. The level of EZH2 was highest at the 
$\mathbf{A}$

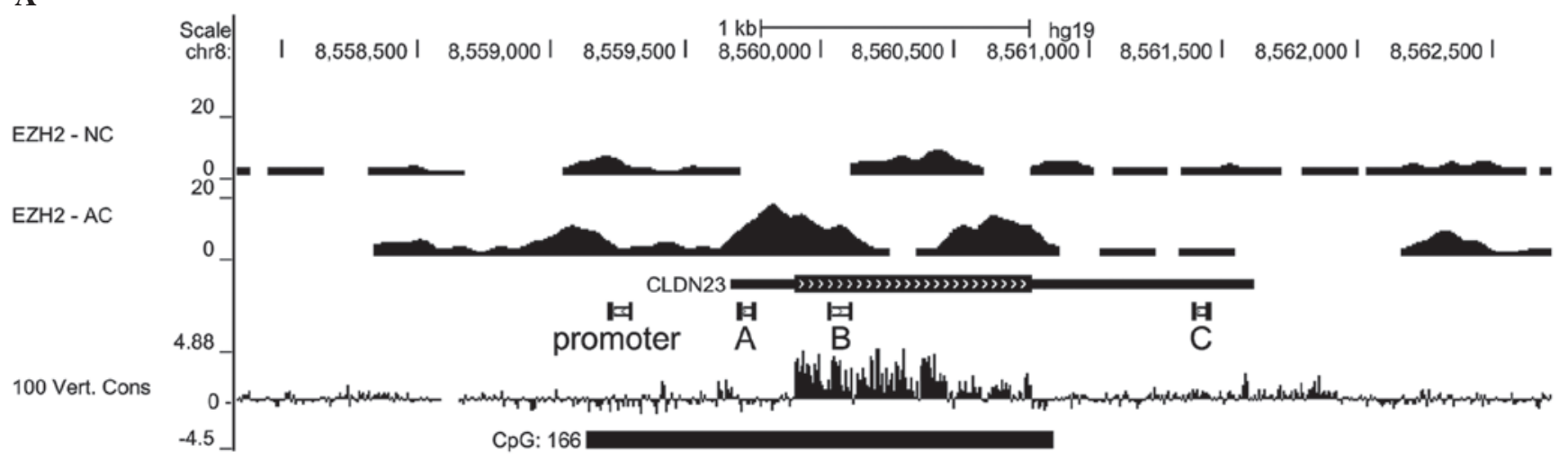

B
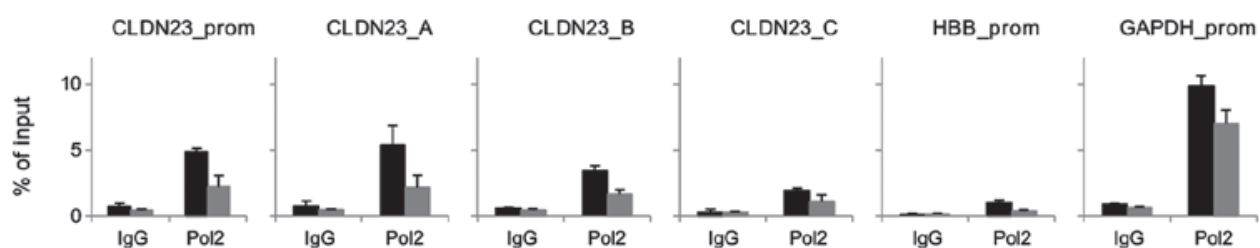

C
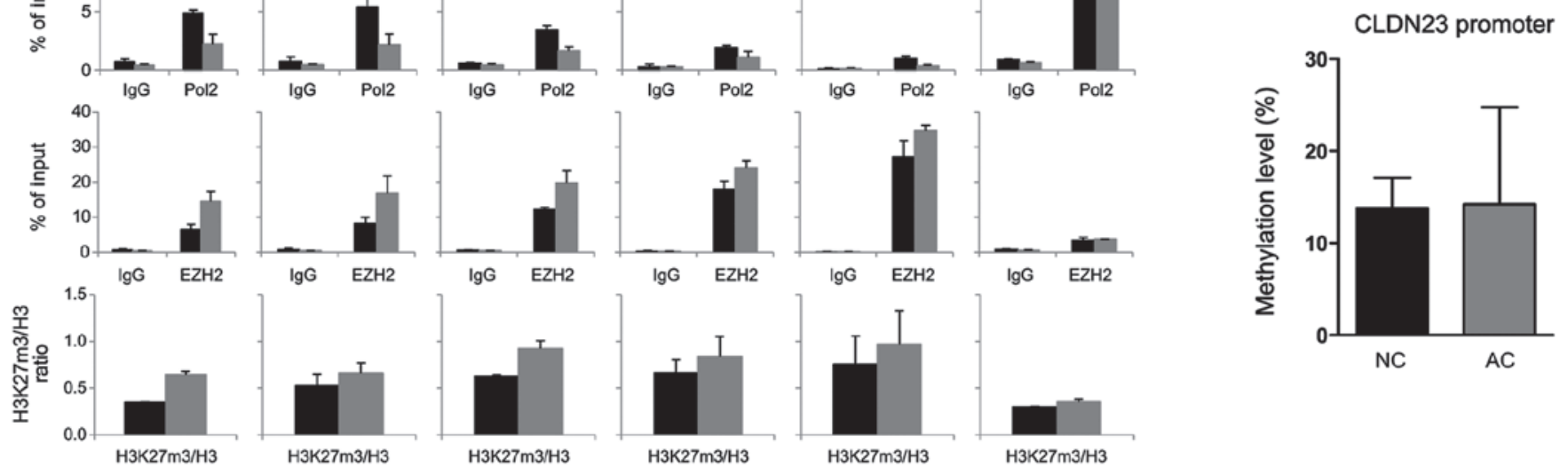

$\lg \mathrm{EZH} 2$

$\mathrm{H} 3 \mathrm{~K} 27 \mathrm{~m} 3 / \mathrm{H} 3$

$\mathrm{H} 3 \mathrm{~K} 27 \mathrm{~m} 3 / \mathrm{H} 3$

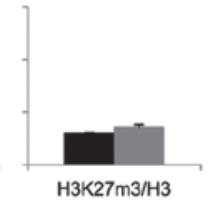

anC $\square \mathrm{AC}$

Figure 2. Binding of EZH2 to the CLDN23 gene is increased in CRC. (A) Chip-Seq EZH2/IgG libraries for pooled AC and NC tissues were sequenced using Genome Analyzer II. Non-specific tags from the IgG library (ChIP assay background) were filtered out. EZH2-specific tags were mapped to the human genome (version hg19) and visualized in the UCSC genome browser. The coordinates of the presented area were chr8:8,557,830-8,562,722. (B) ChIP-qPCR data of the pooled samples used in the ChIP-Seq survey for Pol2, EZH2 and H3K27m3. ChIP results are shown for the PCR products depicted in A. ChIP data are expressed as the DNA recovered, either as the percentage of input DNA, or as the ratio of H3K27m3 to total histone $\mathrm{H} 3$ (mean \pm standard deviation; $\mathrm{n}=3$ ). (C) Pyrosequencing analysis of the methylation of the CLDN23 5' end CpG island in 12 pairs of NC and AC tissue samples. DNA methylation data is expressed as a percentage of fully methylated reference DNA. CRC, colorectal cancer; Ig, immunoglobulin; AC, adenocarcinoma; NC, normal colon; EZH2, enhancer of zeste 2; CLDN23, claudin 23; ChIP, chromatin immunoprecipitation; qPCR, quantitative polymerase chain reaction.

HBB promoter and lowest at the ACTB promoter. The level of Pol2 at CLDN23 remained unchanged upon treatment with GSK126. The level of Pol2 was lowest at the HBB promoter and highest at the ACTB promoter. $\mathrm{H} 3 \mathrm{~K} 4 \mathrm{me} 3$ is associated with the $5^{\prime}$ ends of actively transcribed genes (23). The level of H3K4me3 at CLDN23 was not affected by treatment with GSK126 and, as expected, decreased towards the 3' end of the gene. Notably, ChIP measurements revealed a high level of $\mathrm{H} 3 \mathrm{~K} 4 \mathrm{me} 3$ at the CLDN23 promoter, which reached 83 and $61 \%$ abundance of that mark at the ACTB promoter in Colo205 and HT-29 cells, respectively.

\section{Discussion}

The claudin family of proteins create a junctional protein complex in epithelial and endothelial cells, which maintains cell-to-cell integrity and regulates the diffusion of ions and macromolecules $(5,6)$. Epithelial tight junctions are modulated during epithelial tissue remodeling, wound repair, inflammation and tumorigenesis (24). There are at least 24 claudin proteins, a number of which are upregulated or downregulated in cancer, and these expression changes are tissue-specific (24). Although claudins are important in cancer growth and progression $(4,7)$, relatively little is known about the underlying mechanisms regulating their differential expression. Cancer-associated epigenetic alterations can deregulate the accessibility of DNA during gene transcription, replication, and DNA repair processes, which result in massive deregulation of gene expression during cancer development and progression (25).

In the present study, using a genome-wide ChIP-Seq analysis, it was demonstrated that a high level of EZH2 at the CLDN23 gene was associated with a decreased mRNA level of 
A
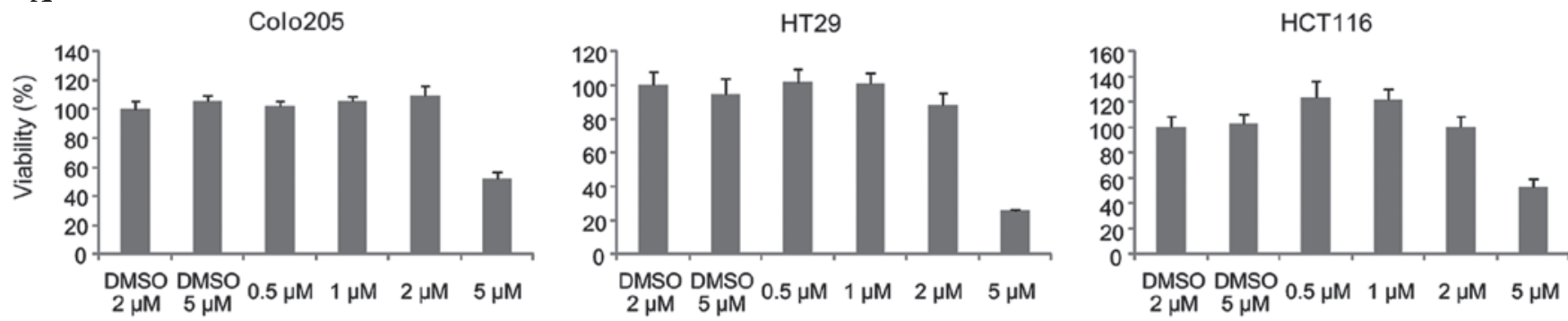

B

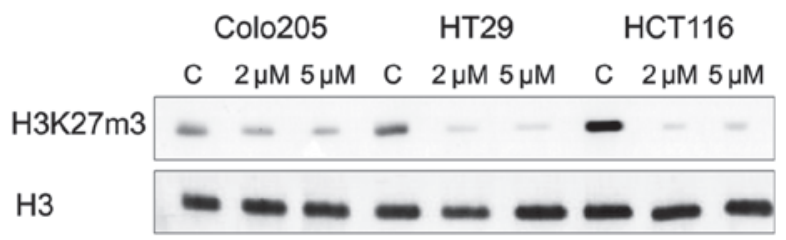

D

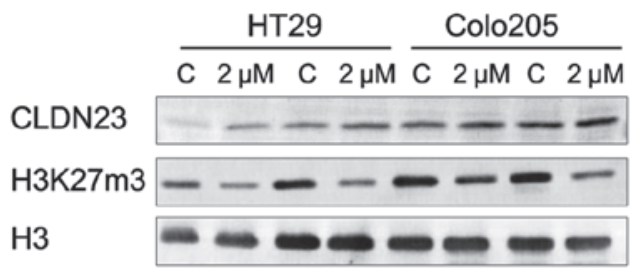

C

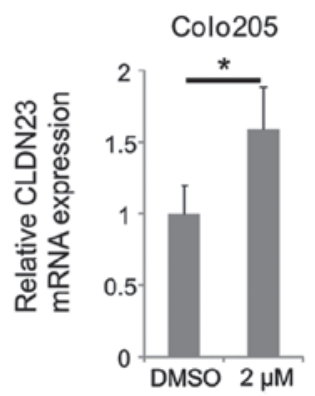

$\mathbf{E}$

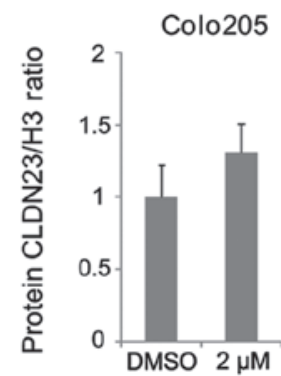

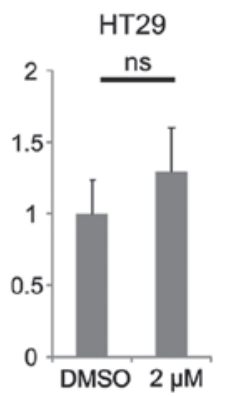

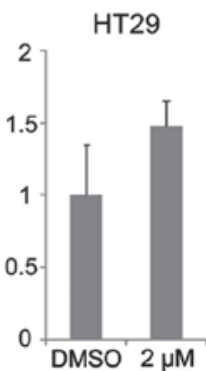

Figure 3. Inhibition of EXH2 by GSK126 increases the expression of CLDN23 in colon cancer cell lines. (A) Colo205, HT-29 and HCT-116 cell lines were cultured under standard conditions in the presence of $0.5,1,2$ or $5 \mu \mathrm{M} \mathrm{GSK} 126$ for $72 \mathrm{~h}$ and were analyzed using a 3-(4,5-dimethylthiazol-2-yl)-2,5-diphenyltetrazolium bromide assay. The data are expressed as percentage viability compared with the control cells maintained in $0.1 \%$ dimethyl sulfoxide (mean \pm standard deviation; n=6). (B) Colo205, HT-29 and HCT-116 cell lines were treated with 2 or $5 \mu$ M GSK126 for $72 \mathrm{~h}$ and subsequently harvested. The lysates were resolved by SDS-PAGE and electrotransferred onto PVDF membranes. Western blot analysis was performed using anti-histone H3 and anti-H3K27me3 antibodies. (C) Colo205 and HCT-116 cell lines were incubated with $2 \mu \mathrm{M}$ GSK126 for $72 \mathrm{~h}$. Total RNA was extracted using TRIzol, treated with DNAse I and subjected to reverse transcription quantitative polymerase chain reaction. The results were normalized against the mRNA expression of RPLP0 (mean \pm standard deviation; $n=4 ;{ }^{*} \mathrm{P}<0.05$ was considered to indicate a statistically significant difference). (D) Colo205 and HCT-116 cell lines were cultured in the presence of $2 \mu \mathrm{M}$ GSK126 for $72 \mathrm{~h}$ and subsequently harvested. Equal quantities of protein were resolved by SDS-PAGE and electrotransferred onto PVDF membranes. Immunostaining was performed using anti-CLDN23, anti-histone H3, and anti-H3K27me3 antibodies. (E) Densities of western blotting bands from (D) were measured using OptiQuant image analysis software. The protein expression level of CLDN23 was normalized to the level of total histone H3 (mean \pm standard deviation; $\mathrm{n}=2$ ). PVDF, polyvinylidene fluoride; EZH2, enhancer of zeste 2; CLDN23, claudin 23.

CLDN23 in CRC tissue (Figs. 1 and 2). The inhibition of EZH2 enzymatic activity using GSK126 increased the protein expression of CLDN23 in HT-29 and Colo205 cell lines (Fig. 3C-E). Analysis of histone modifications upon the inhibition of EZH2 revealed that the level of $\mathrm{H} 3 \mathrm{~K} 27 \mathrm{~m} 3$ was decreased at the CLDN23 promoter, whereas the level of H3K4me3 was high (Fig. 4). The presence of H3K4m3 and H3K27m3 modifications, which are markers of active and repressed transcription, respectively, simultaneously at a promoter was first observed in embryonic stem (ES) cells and these regions were termed 'bivalent domains' (26). Previous studies, using publically available chromatin datasets, also detected bivalent domains in cell types of restricted potency and cancer cell lines $(27,28)$. The observation that genes central to development/embryogenesis exhibit bivalent histone modifications suggested that this bivalency enables regulation of the expression of crucial factors during development and protects against unscheduled gene expression, collectively contributing to the robustness of these processes and reducing transcriptional noise (29). Notably, the CLDN23 gene has been classified as bivalent in three genome-wide analyses of chromatin states in ES cells performed by Mikkelsen et al (30), Ku et al (31) and Brookes et al (32). The latter study demonstrated that the CLDN23 gene is occupied by EZH2 and components of the PRC1 complex, namely, Suz12 and the ubiquitin ligase Ring1B, which monoubiquitinylates histone H2A at K119. Furthermore, this previous study demonstrated that, in ES cells, the CLDN23 promoter is bound by Pol2, which is phosphorylated at Ser5 within its C-terminal domain, and is the form of Pol2, which initiates transcription. The results did not assign CLDN23 to the actively expressed 

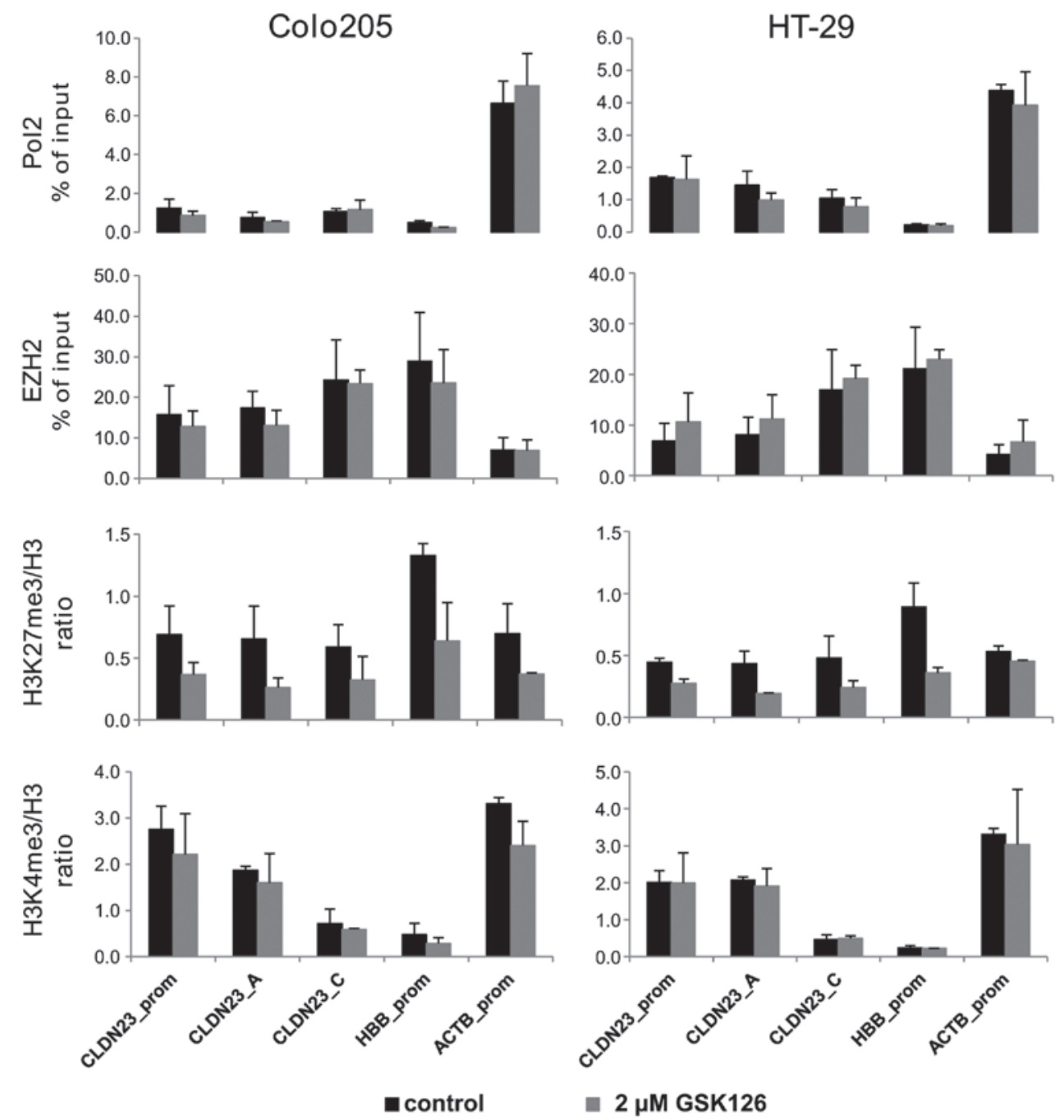

Figure 4. Epigenetic changes at the CLDN23 loci in Colo205 and HT-29 cell lines following treatment with GSK126. Colo205 and HT-29 cell lines were cultured in the presence of $2 \mu \mathrm{M}$ GSK126 for $72 \mathrm{~h}$ and subsequently harvested. Total chromatin was isolated and used in ChIP assays with anti-Pol2, anti-EZH2, anti-histone $\mathrm{H} 3$, anti-H3K4me3 and anti-H3K27me3 antibodies. Isolated DNA was used as the template in reverse transcription quantitative polymerase chain reaction analyses, using primers designed to amplify the CLDN23 gene and the promoters of the HBB and ACTB genes. The data are expressed as a percentage of the input chromatin for Pol 2 and EZH2, or as the ratios of modified histone ChIP signals to total histone H3 ChIP signals. The data are expressed as the mean \pm standard deviation of two independent experiments. EZH2, enhancer of zeste 2; CLDN23, claudin 23; Pol, polymerase; HBB, $\beta$-globin; ACTB, $\beta$-actin.

group of PRC-occupied genes in ES cells, however, the study revealed that even genes exhibiting high levels of expression can be associated with repressive PRCs. This indicated that bivalency represents a dynamic equilibrium between transcription activation and repression, which maintains genes in a plastic, inducible state, enabling increased expression levels when required (29). Of note, bivalent histone modifications also effect the expression of the CLDN4 gene. The expression of CLDN4 is repressed in healthy gastric and ovarian tissues in association with bivalent histone modifications, and the loss of repressive histone methylations and gain of active histone modifications during tumorigenesis correlate with CLDN4 upregulation in gastric (33) and ovarian (34) tissues.

The human CLDN23 gene was first characterized by Katoh and Katoh (35) following the observation that the mRNA encoded by CLDN23 was downregulated in gastric cancer tissue. The CLDN23 transcript was reported to be significantly downregulated in CRC tissue and a lower expression level of this transcript was correlated with shorter overall survival rates in CRC patients (36). Although the CLDN23 gene was first described more than a decade ago, few studies have investigated and no reports have described the mechanism regulating its expression (7). The present study provided evidence that the expression of CLDN23 is epigenetically regulated and disruption of bivalent histone modifications at the CLDN23 locus may be responsible for the substantial downregulation of the expression of CLDN23 in CRC tissue compared with that in NC tissue. It was hypothesized that this downregulation contributes to a decrease or loss of intercellular attachment, providing cancerous cells with more oxygen and nutrients in their microenvironment, enabling them to metastasize from their primary site to other areas of the body. This hypothesis requires further investigation.

\section{Acknowledgements}

This study was supported by a grant from the Ministry of Science and Higher Education (no. N401 071439). 


\section{References}

1. Di Croce L and Helin K: Transcriptional regulation by Polycomb group proteins. Nat Struct Mol Biol 20: 1147-1155, 2013.

2. Chang $\mathrm{CJ}$ and Hung $\mathrm{MC}$ : The role of $\mathrm{EZH} 2$ in tumour progression. Br J Cancer 106: 243-247, 2012.

3. Forbes MS: Cell Structure. In: Cell Physiology Source Book (4th edition). Sperelakis N (ed) Academic Press, San Diego, CA pp67-83, 2012.

4. Ding L, Lu Z, Lu Q and Chen YH: The claudin family of proteins in human malignancy: a clinical perspective. Cancer Manag Res 5: 367-375, 2013.

5. Findley MK and Koval M: Regulation and roles for claudin family tight junction proteins. IUBMB Life 61: 431-437, 2009.

6. Günzel D and Yu ASL: Claudins and the modulation of tight junction permeability. Physiol Rev 93: 525-569, 2013.

7. Turksen K and Troy TC: Junctions gone bad: claudins and loss of the barrier in cancer. Biochim Biophys Acta 1816: 73-79, 2011.

8. Valle BL and Morin PJ: Claudins in Cancer Biology. In: Current Topics in Membranes. Yu ASL (ed.) Vol 65. Academic Press, Amsterdam, pp293-333, 2010.

9. Hanahan D and Weinberg RA: Hallmarks of cancer: the next generation. Cell 144: 646-674, 2011.

10. Frixen UH, Behrens J, Sachs M, et al: E-cadherin-mediated cell-cell adhesion prevents invasiveness of human carcinoma cells. J Cell Biol 113: 173-185, 1991.

11. Hirohashi S and Kanai Y: Cell adhesion system and human cancer morphogenesis. Cancer Sci 94: 575-581, 2003.

12. Skrzypczak M, Goryca K, Rubel T, et al: Modeling oncogenic signaling in colon tumors by multidirectional analyses of microarray data directed for maximization of analytical reliability. PLoS One 5: e13091, 2010.

13. Mikula M, Rubel T, Karczmarski J, Goryca K, Dadlez M and Ostrowski J: Integrating proteomic and transcriptomic high-throughput surveys for search of new biomarkers of colon tumors. Funct Integr Genomics 11: 215-224, 2011.

14. Flanagin S, Nelson JD, Castner DG, Denisenko O and Bomsztyk K: Microplate-based chromatin immunoprecipitation method, Matrix ChIP: a platform to study signaling of complex genomic events. Nucleic Acids Res 36: e17, 2008.

15. Yu J, Feng Q, Ruan Y, Komers R, Kiviat N and Bomsztyk K: Microplate-based platform for combined chromatin and DNA methylation immunoprecipitation assays. BMC Mol Biol 12: 49, 2011.

16. Mikula M, Bomsztyk K, Goryca K, Chojnowski K and Ostrowski J: Heterogeneous nuclear ribonucleoprotein (HnRNP) K genome-wide binding survey reveals its role in regulating 3 '-end RNA processing and transcription termination at the early growth response 1 (EGR1) gene through XRN2 exonuclease. J Biol Chem 288: 24788-24798, 2013.

17. Aird D, Ross MG, Chen W-S, et al: Analyzing and minimizing PCR amplification bias in Illumina sequencing libraries. Genome Biol 12: R18, 2011.
18. Langmead B, Trapnell C, Pop M and Salzberg SL: Ultrafast and memory-efficient alignment of short DNA sequences to the human genome. Genome Biol 10: R25, 2009.

19. Zhang Y, Liu T, Meyer CA, et al: Model-based analysis of ChIP-Seq (MACS). Genome Biol 9: R137, 2008.

20. Kent WJ, Sugnet CW, Furey TS, et al: The human genome browser at UCSC. Genome Res 12: 996-1006, 2002.

21. ENCODE Project Consortium: An integrated encyclopedia of DNA elements in the human genome. Nature 489: 57-74, 2012.

22. McCabe MT, Ott HM, Ganji G, et al: EZH2 inhibition as a therapeutic strategy for lymphoma with EZH2-activating mutations. Nature 492: 108-112, 2012.

23. Mikula M, Gaj P, Dzwonek K, et al: Comprehensive analysis of the palindromic motif TCTCGCGAGA: a regulatory element of the HNRNPK promoter. DNA Res 17: 245-260, 2010.

24. Singh AB, Sharma A and Dhawan P: Claudin family of proteins and cancer: an overview. J Oncol 2010: 2010.

25. Chi P, Allis CD and Wang GG: Covalent histone modifications - miswritten, misinterpreted and mis-erased in human cancers. Nat Rev Cancer 10: 457-469, 2010.

26. Bernstein BE, Mikkelsen TS, Xie X, et al: A bivalent chromatin structure marks key developmental genes in embryonic stem cells. Cell 125: 315-326, 2006.

27. Larson JL and Yuan GC: Chromatin states accurately classify cell differentiation stages. PLoS One 7: e31414, 2012.

28. Rodriguez J, Muñoz M, Vives L, Frangou CG, Groudine M and Peinado MA: Bivalent domains enforce transcriptional memory of DNA methylated genes in cancer cells. Proc Natl Acad Sci USA 105: 19809-19814, 2008.

29. Voigt P, Tee WW and Reinberg D: A double take on bivalent promoters. Genes Dev 27: 1318-1338, 2013.

30. Mikkelsen TS, Ku M, Jaffe DB, et al: Genome-wide maps of chromatin state in pluripotent and lineage-committed cells. Nature 448: 553-560, 2007.

31. Ku M, Koche RP, Rheinbay E, et al: Genomewide analysis of PRC1 and PRC2 occupancy identifies two classes of bivalent domains. PLoS Genet 4: e1000242, 2008.

32. Brookes E, de Santiago I, Hebenstreit D, et al: Polycomb associates genome-wide with a specific RNA polymerase II variant, and regulates metabolic genes in ESCs. Cell Stem Cell 10: $157-170,2012$.

33. Kwon MJ, Kim SH, Jeong HM, et al: Claudin-4 overexpression is associated with epigenetic derepression in gastric carcinoma. Lab Invest 91: 1652-1667, 2011.

34. Kwon MJ, Kim SS, Choi YL, et al: Derepression of CLDN3 and CLDN4 during ovarian tumorigenesis is associated with loss of repressive histone modifications. Carcinogenesis 31: 974-983, 2010.

35. Katoh M and Katoh M: CLDN23 gene, frequently down-regulated in intestinal-type gastric cancer, is a novel member of CLAUDIN gene family. Int J Mol Med 11: 683-689, 2003.

36. Pitule P, Vycital O, Bruha J, et al: Differential expression and prognostic role of selected genes in colorectal cancer patients. Anticancer Res 33: 4855-4865, 2013. 